\title{
Evaluación de la expansividad de los suelos en el reparto Hermanos Cruz, Pinar del Río, Cuba: una contribución a la planificación urbana
}

\author{
G.A. Chinthaka-Ganepola(1), Abdo Said Mohammed(1), Alexis Ordaz Hernández ${ }^{(2)}$, Elmidio Estévez \\ Cruz $^{(1)}$ y José Ramón Hernández Santana(3) \\ (1) Departamento de Geología, Universidad de Pinar del Río, calle Martí 270 Final, C.P. 20100, Pinar del Río, Cuba. \\ gacganepola@gmail.com \\ abdos_955@yahoo.com \\ elmidio2013@gmail.com \\ (2) Facultad de Geografía, Universidad Autónoma del Estado de México, Paseo Universidad, Ciudad Universitaria, \\ C.P. 50110, Toluca de Lerdo, México. \\ ordaz1978@ hispavista.com \\ (3) Instituto de Geografía, Universidad Nacional Autónoma de México, Ciudad Universitaria, \\ C.P. 04510, Coyoacán, México, D.F. \\ santana@igg.unam.mx
}

\section{RESUMEN}

Los suelos expansivos constituyen un peligro geológico, muchas veces desconocido para la población, que puede afectar a las edificaciones y las infraestructuras. Para evaluar esta problemática del suelo, es necesario conocer algunas de sus propiedades geotécnicas. Generalmente, en las metodologías diseñadas para este fin se emplean valores promedios de las propiedades de los suelos, de la formación geológica o grupo litológico, aspecto que conlleva una gran incertidumbre. Para superar este problema, en la investigación se propone un procedimiento para la estimación espacial y modelación de las principales propiedades del suelo que inciden en la evaluación de su expansividad. El caso de estudio seleccionado comprende la localidad Hermanos Cruz, perteneciente a la Ciudad de Pinar del Río en el occidente de Cuba. La investigación se organizó en las siguientes etapas: evaluación primaria del potencial de expansión de los suelos, modelación de propiedades de los suelos utilizando los métodos geoestadísticos en 3D y, finalmente, representación cartográfica del potencial de expansión de los suelos sobre plataforma SIG a profundidades del subsuelo con importancia en la construcción de las cimentaciones superficiales de las obras ingenieriles. La aplicación de este procedimiento al caso de estudio seleccionado reveló que los suelos del reparto Hermanos Cruz poseen un potencial de expansión bajo a medio y la existencia de sectores aislados con un potencial de expansión medio a alto.

Palabras clave: expansividad, geoestadística, propiedades de los suelos arcillosos

\section{Evaluation of the potential expansiveness of soils in the Hermanos Cruz neighbourhood, Pinar del Rio, Cuba: a contribution to urban planning}

\begin{abstract}
Expansive soils can be categorized as one of the geohazards observed in the urban environment, representing a silent hazard to buildings and infrastructure. To evaluate this problematic soil it is necessary to know some of its geotechnical properties. Generally, the average values of soil properties are used in the methods which characterize the geological formation and the lithological group, which causes a great degree of uncertainty. To overcome this problem, this study proposes a procedure for estimating and modelling the principle soil properties that have an impact on expansive soils. The selected case study is located in the Hermanos Cruz neighbourhood in the city of Pinar del Rio in Cuba. The investigation was organized into the following stages: primary assessment of the potential expansiveness of the soils, modelling of the soil properties utilizing $3 D$ geostatistical methods and finally the cartographic representation of the potential
\end{abstract}


expansiveness of soils on a Geographic Information System (GIS) platform at different depth zones which are of importance in the construction of shallow foundations of engineering works. The application of this methodology in the Hermanos Cruz neighbourhood revealed that its soils possess a potential expansiveness of low to medium, apart from some isolated zones which show a potential expansiveness of medium to high.

Keywords: expansiveness, geostatistics, clay soils properties

\section{ABRIDGED ENGLISH VERSION}

\section{Introduction}

The presence of expansive soils is often a silent hazard to the population and infrastructure. Soils of this nature mainly affect lightweight structures, such as roadways and single storey buildings. Expansive soils exhibit volumetric changes in response to variations in moisture content and internal stresses caused by water molecules in the soils. Various swelling mechanisms described in the literature (Sowers and Sowers 1987; Delgado 2003), are classified into three major groups namely: electrical repulsion of clay particles, osmotic repulsion and hydration of cations. Other mechanisms of minor influence are the Van der Waals forces and the elastic forces between clay particles.

According to geotechnical specialists of the National Enterprise for Applied Research (ENIA) in Cuba, $49 \%$ of the building pathologies of the city of Pinar del Río are caused by the existence of expansive soils. Damage occurring in these structures includes cracks in building walls, foundations and roadways. Girbert (2015) describes the presence of expansive clays with thicknesses of up to $5.0 \mathrm{~m}$, in the northeast of the city in the Hermanos Cruz neighbourhood (Fig. 1).

Currently, there are significant classification criteria (Snethen 1980 in: Djedid and Bekkouche 2001; Seed et al., 1962 in: Djedid and Bekkouche 2001; Building Research Establishment 1980 in: Djedid and Bekkouche 2001 and Holtz et al., 1973 in: Djedid y Bekkouche 2001), classification charts (abacus) (Seed et al., 1960 in: Lucian 2006; Van Der Merwe 1964 in: Lucian 2006 and Skempton 1953 in: Lucian 2006) and empirical formulae (Delgado 2003 and Martínez 2012), which define the potential expansiveness of soils from geotechnical parameters.

This study utilizes the above mentioned criteria and incorporates the use of $3 D$ geostatistical estimation methods, to present a cartographic representation of the potential expansiveness of soils with zones of different expansive levels in the Hermanos Cruz neighbourhood which is the principle objective of this paper.

\section{Methods}

The study was carried out in the Hermanos Cruz neighbourhood located in the city of Pinar del Rio in the following stages: (1) primary assessment of the potential expansiveness of soils, (2) modelling of soil properties utilizing $3 D$ geostatistical methods and (3) cartographic representation of the potential expansiveness of soils on the GIS platform at different depth zones.

\section{Results and discussion}

(1) Primary assessment of the potential expansiveness of soils:

To characterize expansive soils, the authors used the following index properties: liquid limit, plasticity index, shrinkage limit and clay content. The data were obtained from the Geotechnical Properties Database of the National Enterprise for Applied Research (ENIA) of Pinar del Rio. The results of the statistical analysis of the given index properties are presented in Table 3. The potential expansiveness of soils was evaluated by applying the classification criteria of Snethen (1980) in: Djedid y Bekkouche (2001); Seed et al. (1962) in: Djedid y Bekkouche (2001); Building Research Establishment (1980) in: Djedid and Bekkouche (2001) and Holtz et al. (1973) in: Djedid and Bekkouche (2001) based on the mean values of the index properties. Classification charts (the Casagrande plasticity chart and Seed et al., 1960 in: Lucian 2006) and empirical formulae (Delgado 2003 and Martínez 2012) were also used in the primary assessment of the potential expansiveness of soils.

The results obtained through these methods are very similar and we were able to safely conclude that the soils of the Hermanos Cruz neighbourhood have a "medium degree" of potential expansiveness. However, it is difficult to determine the spatial variation of volumetric changes of soils. Therefore, another method of examining the data, such as modelling of soil properties utilizing 3D geostatistical methods, has been adopted. 
(2) Modelling of soil properties utilizing 3D geostatistical methods:

In this stage, firstly the geological 3D model (Fig. 5) was generated by identifying engineering geological

homogeneous units namely:

- Vegetation / fill layer: the superficial deposits found in the study area.

- Marine alluvial coarse-grained soil: These soils consist of sands, loamy sands and sandy gravels classified according to the Unified Soil Classification System (USCS) as clayey sand (SC) and clayey gravels (GC). This unit coincides with the sandy phase of the Guane Formation.

- Marine alluvial fine grained soil: Composed mainly of fine grained soils which can be classified as clays of high plasticity $(\mathrm{CH})$ and clays of low plasticity $(\mathrm{CL})$. This unit coincides with the clay phase of the Guane Formation.

- Eluvial soils: These soils or highly weathered rocks which are found in the soil state belonging to the top layer of the Paso Real Formation. This unit mainly consists of carbonated clay soils with limestone fragments. Its soils are classified as clayey sand soils (SC) and clays of low plasticity (CL).

- Bed Rock: This unit forms the basal part of the study area. It consists of alternating limestone and marl stones.

The Triangular Irregular Networks (TIN) algorithm was used to build up the 3D geometric model. Index properties of the soils were then estimated in the $3 D$ space bounded by the geometrical limits of the engineering geological units.

According to the geological model, marine alluvial coarse-grained soils and marine alluvial fine grained soils form the greater part of the top sub-soil layer, where clays of low and high plasticity and clayey sand are found in abundance.

Subsequently, the geomechanical models of the index parameters (clay content, plasticity index and liquid limit) were elaborated. To achieve this, the index parameters were estimated using 3D Kriging Ordinary algorithm within the geometric boundaries defined by the geological model. The elaborated geomechanical models are shown in Figures 8, 9 and 10 and they provide valuable information for integrated data analysis, in order to establish the spatial variation of the potential expansiveness of soils on the GIS platform.

(3) Cartographic representation of the potential expansiveness of soils on the GIS platform at different depth zones which are of major engineering importance:

The estimated results of clay content, plasticity index and liquid limit obtained by $3 D$ geostatistics were transferred to GIS. They were then reclassified into three levels of potential expansiveness; low, low to medium and medium to high, and finally combined together on the GIS platform in order to generate a cartographic representation of the potential expansiveness of soils at different depth zones which are of major engineering importance.

The resulting potential expansiveness maps are shown in Figures 11, 12 and 13.

These maps show the existence of isolated areas where the potential expansiveness is medium to high. In these areas the soil column is composed predominantly of clay soils classified as clays of high and low plasticity (CH and CL). A major portion of the Study area is characterized by soils with a potential expansiveness of low to medium.

Finally the potential expansiveness maps were subjected to a validation process. The analysis was performed by overlaying the potential expansiveness map on the map of damages to infrastructure due to expansive soils (Fig.14). Thus the spatial coincidence between the locations of the damage and the critical areas shown by the above maps was found.

\section{Conclusions}

1. The use of classification charts and empirical formulae allowed a primary assessment of the potential expansiveness of soils in the Hermanos Cruz neighbourhood, showing a "medium" degree of potential expansiveness of soils, but did reveal the spatial variation of volumetric changes.

2. The geological model of the subsoil layer on which the Hermanos Cruz neighbourhood has been located, is defined by the presence of five engineering geological homogeneous units namely: vegetation/fill layer, marine alluvial coarse grained soil, marine alluvial fine grained soil, eluvial soil and bed rock.

3. Integration of results of the modelling of geotechnical properties, together with quantitative methods, enabled the cartographic representation of the potential expansiveness of soils at different depth zones on the GIS platform. This information is vital for sustainable urban planning practices in the Hermanos Cruz neighbourhood. 
4. The application of this method reveals that the soils of Hermanos Cruz neighbourhood exhibit a potential expansiveness of low to medium and the existence of isolated sectors with a potential expansiveness of medium to high.

\section{Introducción}

La presencia de suelos expansivos representa un riesgo muchas veces desconocido para la población. El fenómeno de la expansividad afecta principalmente a las estructuras que transmiten presiones bajas al terreno, como pavimentos y edificaciones ligeras. EI fenómeno se fundamenta en los cambios volumétricos ocasionados por las variaciones en la humedad y por los esfuerzos internos producidos por el agua. Los diversos mecanismos de expansión descritos en la literatura técnica (Sowers y Sowers 1987; Delgado 2003) se agrupan en tres importantes grupos: repulsión eléctrica de las partículas de arcilla, repulsión osmótica e hidratación de cationes. Otros mecanismos de menor influencia en la expansión son las fuerzas de Van der Waals y la relajación elástica.

Los riesgos causados por suelos expansivos poseen características peculiares, pues no producen pérdidas de vidas humanas y los daños en las construcciones se perciben muy espaciados en el tiempo. Como consecuencia del impacto de dicho fenómeno en el ambiente urbano, se generan importantes pérdidas económicas. En Londres (Inglaterra), por ejemplo, los daños a las cimentaciones llegan a ser $£ 500$ millones por año debido a la presencia de las arciIlas expansivas (Jones y Terrington 2011); en Estados Unidos los suelos expansivos causan daños a edificios, aeropuertos, tuberías, entre otras estructuras, por aproximadamente 9.000 millones de dólares cada año, más del doble de las pérdidas por los llamados "desastres naturales" (Bimbela 2014). De estos y muchos otros ejemplos, se deriva la importancia del estudio detallado de este fenómeno geotécnico.

Según los datos de la Empresa Nacional de Investigaciones Aplicadas (ENIA) de Cuba, el 49\% de las construcciones con estudios de patologías constructivas de la ciudad de Pinar del Río, experimentan daños por cambios volumétricos del suelo por variación de la humedad. Estos cambios se reflejan en los muros de los edificios y en las carreteras, ocasionando grietas. Girbert (2015) señala la existencia de arcillas expansivas con espesores de hasta $5.0 \mathrm{~m}$ medidos desde la superficie, en el noreste de la ciudad en el reparto Hermanos Cruz (Figura 1).

Los estudios sobre el potencial de expansividad del subsuelo se centran en evaluar sus propiedades intrínsecas, tales como el tipo de mineral arcilloso y las propiedades del fluido de los poros, las que dependen de la composición electroquímica del agua y la interacción agua-arcilla (Delgado 2003). Las propiedades citadas tienen una relación directa con el comportamiento geotécnico de los suelos, que puede ser evaluado mediante algunas propiedades físicas o mecánicas.

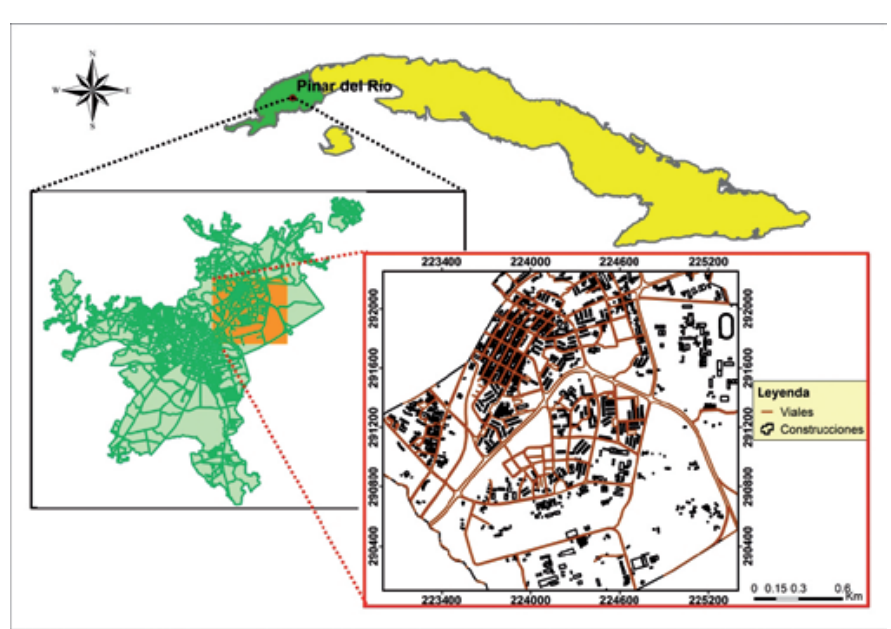

Figura 1. Situación geográfica del área de estudio.

Figure 1. Geographical location of the study area.

En la actualidad, existe una serie de criterios (Seed et al. 1962 en: Djedid y Bekkouche 2000; Snethen 1980 en: Djedid y Bekkouche 2001; Building Research Establishment 1980 en: Djedid y Bekkouche 2001 and Holtz et al. 1973 en: Djedid y Bekkouche 2001), juegos de curvas (ábacos) (Skempton 1953 en: Lucian 2006; Seed et al. 1960 en: Lucian 2006 y Van Der Merwe 1964 en: Lucian 2006) y ecuaciones empíricas (Delgado 2003 y Martínez 2012), enfocados a definir el potencial de expansión de los suelos a partir de sus propiedades físicas. Este trabajo aplica estas experiencias, e incorpora el empleo de los métodos geoestadísticos de estimación, aportando rigor a los resultados.

Los métodos geoestadísticos de estimación se fundamentan en cuantificar la continuidad espacial y emplear la misma en el proceso de estimación, tradicionalmente empleados en trabajos de prospección minera. Recientemente, otras ramas de las geociencias han incorporado esta herramienta, especialmente en la solución de problemas geotécnicos (Bourgine et al. 2006; Breysse et al. 2006; Mendes 
y Lorandi 2006; Choi y Park 2006). En el caso de la República de Cuba, la aplicación de las técnicas de estimación geoestadísticas en el campo de la geotecnia, han sido muy limitadas (Rivera 2013; Ordaz et al. 2014; Ordaz 2014), lo que le confiere un valor particular a los resultados obtenidos en esta investigación.

Con el presente trabajo se pretende realizar una aportación en la zonificación de las áreas con presencia de suelos proclives a experimentar cambios volumétricos, que en la actualidad afectan a las infraestructuras del caso de estudio seleccionado. El resultado final constituiría una herramienta de consulta para el ordenamiento urbano de la ciudad y además incentiva el empleo de la información geotécnica con fines de evaluación de peligros geológicos en entornos urbanos.

\section{Condiciones geológicas}

La ciudad de Pinar del Río se asienta sobre los sedimentos de la cuenca cenozoica Los Palacios. Esta estructura deprimida es un monoclinal que buza hacia el sur, con un ángulo muy suave de $7^{\circ}$ a $8^{\circ}$, cuya columna estratigráfica está constituida por rocas vulcano-sedimentarias del Cretácico Inferior (Aptiano - Albiano), que yacen discordantemente sobre un basamento ofiolítico. Este yace a unos $3 \mathrm{~km}$ de profundidad y está conformado por restos de corteza oceánica, representados por un complejo ultramáfico de gabros, serpentinitas, basaltos y otras rocas.

Sobre el complejo vulcano-sedimentario se depositaron discordantemente los sedimentos terrígenos-carbonatados de la Formación Los Negros del Cretácico Superior (Campaniano-Maestrichtiano), con un espesor de 500-700 m, que incluyen areniscas, limolitas polimícticas y vulcanomícticas, con intercalaciones de gravelitas y conglomerados vulcanomícticos y calizas micríticas con rudistas (Cabrera et al. 2004).

Posteriormente, sobre estos últimos sedimentos se depositaron discordantemente las formaciones geológicas cenozoicas, de composición terrígeno-carbonatadas, con un buzamiento horizontal y sub-horizontal y edades desde el Eoceno Medio al Mioceno Medio. Específicamente, para el reparto Hermanos Cruz (Caso de estudio) en algunos sectores afloran las rocas cenozoicas de la Formación Paso Real. Hacia el Plioceno Superior comienza una transgresión marina originándose una llanura aluvial y aluvial marina, este régimen de sedimentación se mantiene hasta el Pleistoceno Superior, donde se depositaron los sedimentos terrígenos la Formación Guane.
La Formación Guane constituye la principal formación arcillosa que se estudia en este trabajo y aflora en el $82 \%$ de la superficie del reparto Hermanos Cruz. Desde el punto de vista litológico está compuesta por arenas y arenas arcillosas de génesis aluvial, asociada geomorfológicamente a terrazas de ríos o a sedimentos de origen aluvial marino. Los ensayos mineralógicos consultados en Girbert (2014) correspondientes a cinco puntos de monitoreo dentro de esta formación geológica, demostraron la presencia de la fase mineral caolinita. La presencia de estos suelos unida a las continuas sucesiones de periodos secos y húmedos, donde las precipitaciones en los meses de Noviembre a Abril no superan los $40 \mathrm{~mm}$ mensuales y en los meses de Mayo a Octubre exceden los $240 \mathrm{~mm}$, son la causa principal de los cambios volumétricos en este caso de estudio.

Desde el punto de vista hidrogeológico la fase arcillosa de la Formación Guane forma el sello protector del horizonte acuífero de los depósitos de la Formación Paso Real, a la que sobreyace en gran parte del área, mientras que la fase arenosa constituye reservorios de agua (lentejones colgados), con variaciones continuas de sus niveles piezométricos en función de los cambios climáticos anuales.

\section{Metodología}

La investigación se organizó en tres etapas:

(1) Evaluación primaria del potencial de expansión de los suelos en el reparto Hermanos Cruz.

(2) Modelación de las propiedades de los suelos.

(3) Representación cartográfica del potencial de expansión de los suelos sobre plataforma SIG.

(1) Para la evaluación primaria del potencial de expansión de los suelos en el reparto Hermanos Cruz, inicialmente fue necesario realizar una base de datos de las propiedades de los suelos que influyen en su expansividad. En este caso se empleó la información proveniente de 655 perforaciones realizadas en el área en los últimos 50 años y almacenada en los archivos técnicos de la Empresa Nacional de Investigaciones Aplicadas de Cuba (Unidad de investigaciones para la construcción de Pinar del Río). La Figura 2 muestra la distribución espacial de los sondeos analizados.

Principalmente se tabularon las siguientes propiedades índice: límite líquido, índice de plasticidad, límite de retracción y contenido de arcilla. Seguidamente, se realizó la estadística descriptiva de estas propiedades que incluye el número de valores, mínimo, 1er cuartil, mediana, 3er cuartil, 
máximo, media, moda, asimetría, curtosis y coeficiente de variación. Posteriormente se aplicaron los criterios de Seed et al. 1962 en: Djedid y Bekkouche 2001; Holtz et al. 1973 en: Djedid y Bekkouche 2001; Snethen 1980 en: Djedid y Bekkouche 2001 y Building Research Establishment 1980 en: Djedid y Bekkouche 2001; además en esta etapa inicial se evaluó la expansividad de los suelos mediante curvas (ábacos) y ecuaciones empíricas (Seed et al. 1960 en: Lucian 2006; Delgado 2003 y Martínez 2012).

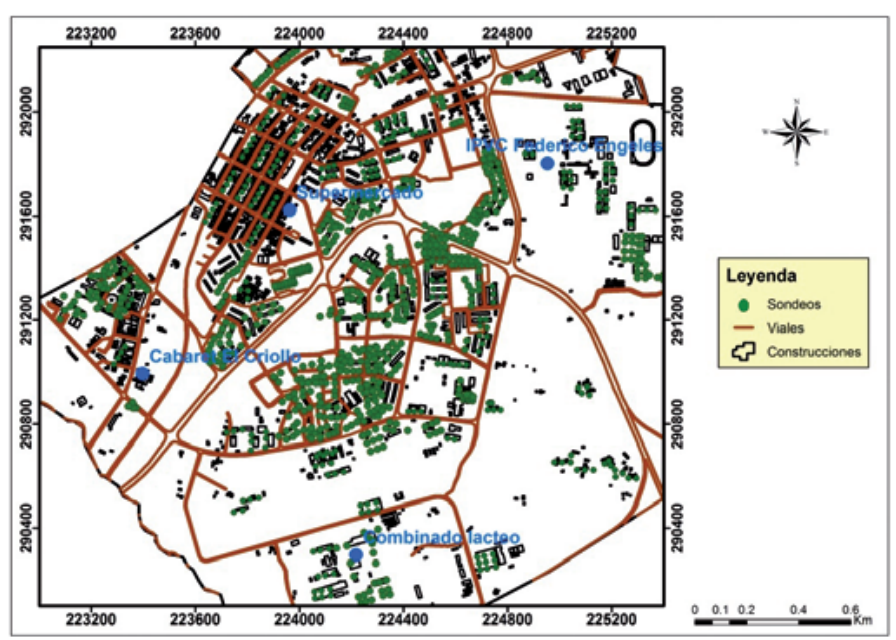

Figura 2. Mapa con la distribución espacial de los sondeos analizados. Escala original 1:25000.

Figure 2. Map showing the spatial distribution of the analyzed borehole locations. Original scale 1:25000.

Los métodos empleados en esta primera etapa, no se alejan de los métodos tradicionalmente empleados para el estudio de problemas vinculados a las arcillas expansivas. El principal aporte hasta aquí se enfoca en develar la presencia de suelos proclives a cambios volumétricos. Para lograrlo se analizaron un total de 942 muestras provenientes de 655 perforaciones, distribuidas en el área objeto de investigación. De esta forma se obtuvo un criterio inicial del comportamiento aproximado y del potencial de expansión de los suelos del reparto Hermanos Cruz, este resultado justifica la sucesión de las etapas posteriores de esta investigación.

Los métodos utilizados en esta primera etapa son insuficientes para revelar el comportamiento espacial del fenómeno estudiado e impide la zonificación de las áreas con presencia de suelos proclives a experimentar cambios volumétricos (objetivo de este trabajo). Como consecuencia la herramienta aportada a los planificadores y tomadores de decisiones tendría limitaciones. Para superar este inconveniente se procedió a la modelación 3D de las propiedades de los suelos y posteriormente a la representación cartográfica del potencial de expansión sobre plataforma SIG, en este caso a profundidades del subsuelo con importancia en el diseño de cimentaciones superficiales. El algoritmo que se describirá a continuación, constituye la primera aplicación de los métodos geoestadísticos en 3D a la solución de problemas geotécnicos en Cuba.

(2) La modelación de las propiedades de los suelos partió de la elaboración del modelo geológico del área de estudio, identificando espacialmente los tipos ingeniero-geológicos (tipos de suelos con propiedades geotécnicas similares). Con el empleo de la base de datos, se genera un modelo geométrico tridimensional, en este caso, empleando el algoritmo de TIN (redes irregulares de triángulos). Posteriormente se modelan las propiedades geotécnicas dentro de los tipos "ingeniero-geológicos" (modelo geomecánico) siguiendo cinco pasos:

(a) Elaboración de bases de datos. La generación del modelo geomecánico demandó la elaboración de una base de datos específica, la cual incluye coordenadas Lambert y la cota de la boca de los pozos $(X, Y$, $Z$ ), la profundidad y el valor de la propiedad geotécnica de interés a diferentes profundidades.

(b) El análisis exploratorio de datos. Consistió en el estudio del comportamiento estadístico de las propiedades de los suelos dentro del modelo geológico elaborado anteriormente. Para lograr este objetivo, se construyeron los histogramas, y de las interpretaciones de los mismos se han obtenido las conclusiones sobre el tipo de distribución que siguen los datos, la presencia de valores errados y extremos, y la posible existencia de poblaciones complejas (bimodalidad o multimodalidad). Esta etapa es de gran importancia, pues facilita la toma de decisiones acerca de las posibles técnicas de interpolación que se deben usar en el momento de la estimación.

(c) El estudio variográfico. Estuvo dirigido a estudiar y cuantificar la variabilidad espacial de cada propiedad analizada, para lo cual fue necesario el cálculo del semivariograma experimental y el ajuste de un modelo teórico conocido.

El cálculo del semivariograma experimental es la herramienta geoestadística más importante en la determinación de las características de variabilidad y correlación espacial de la propiedad geotécnica estudiada. Se construyeron los semivariogramas experimentales en cuatro direcciones con azimut $0^{\circ}, 45^{\circ}$, $90^{\circ}$ y $135^{\circ}$, y de esta forma se analizó la presencia de anisotropía en la variable analizada. Para el cálculo 
de los semivariogramas experimentales en la investigación se utilizó un incremento o paso de $20.0 \mathrm{~m}$ que coincide con el espaciamiento promedio entre las perforaciones, la tolerancia lineal fue la mitad del incremento inicial y el cálculo se realizó hasta aproximadamente 10 puntos en el semivariograma, es decir hasta $200.0 \mathrm{~m}$, distancia suficiente para estudiar la variabilidad espacial que presentan las variables analizadas.

En la dirección vertical, los variogramas fueron calculados usando un paso de $1.0 \mathrm{~m}$, que coincide con la separación promedio de las muestras tomadas durante la documentación geotécnica. Luego se ajusta a un modelo teórico con el objetivo de determinar los parámetros descriptivos del semivariograma que posteriormente serán usados en la estimación.

(d) Estimación de las propiedades de los suelos. El método empleado para la estimación de las propiedades de los suelos de interés fue el modelo de bloques, el cual consiste en la discretización del espacio 3D en bloques o celdas tridimensionales (voxels), que contiene los atributos (contenido de arcilla, índice de plasticidad y límite líquido). Las dimensiones de bloques $(25 \mathrm{~m} \times 25 \mathrm{~m} \times 1 \mathrm{~m})$ se seleccionaron considerando el espaciamiento promedio entre las perforaciones. Como método de interpolación se utilizó el Krigeaje Ordinario (Kriging Ordinario). Este método considera que la media local no está necesariamente cercana a la media de la población, usa solamente en el estimado la muestra para la vecindad local (Cuador et al. 1997). La vecindad de búsqueda se diseñó de manera que los interpoladores espaciales emplearan las muestras más cercanas al bloque, evitando que las mismas procedieran únicamente de un solo sondeo o sector alrededor del bloque. Finalmente se calcula el grado de incertidumbre del modelo geomecánico confeccionado a través de la función varianza krigeaje.

Los procesos (b), (c) y (d) se realizaron utilizando el software de modelación geoestadística Stanford Geostatistical Modeling Software (SGeMS).

(e) Creación del modelo cuantitativo. En este paso a partir de los datos estimados se confeccionó el modelo geomecánico final. Posteriormente, se pueden construir perfiles y vistas en plantas donde se visualizan los resultados del modelo.

(3) Para la representación cartográfica del potencial de expansión de los suelos sobre la plataforma SIG, se eligieron tres intervalos de profundidad: 0 a $1.0 \mathrm{~m}, 1.0$ a $2.0 \mathrm{~m}$ y de 2.0 a $3.0 \mathrm{~m}$, todos de interés para las cimentaciones superficiales. Primeramente se confeccionaron los mapas temáticos tomando los valores de las propiedades de los suelos: contenido de arcilla, índice de plasticidad y límite líquido, para cada zona de profundidad anteriormente indicada. Posteriormente se reclasificaron los tres mapas atendiendo a tres niveles del potencial de la expansión para cada propiedad: bajo, bajo a medio y medio a alto. Los rangos adoptados en este caso de estudio fueron sugeridos por los autores citados en la Tabla 1.

Por último, se combinan los tres mapas temáticos, para delimitar las áreas coincidentes con las mismas condiciones del potencial de expansión (Tabla 2). Como resultado final se generó un mapa del potencial de expansión de los suelos para cada zona de profundidad anteriormente descrita. Todo el proceso se llevó a cabo sobre plataforma de sistema de información geográfica.

\section{Resultados y discusión}

\section{Evaluación primaria del potencial de expansión de los suelos en el reparto Hermanos Cruz}

Para la caracterización de los suelos expansivos, los autores utilizaron las siguientes propiedades índices: límite líquido, índice de plasticidad, límite de retracción y el contenido de arcilla. Los resultados del análisis estadístico se presentan en la Tabla 3.

Considerando "la media" de las diferentes propiedades índices plasmadas en la Tabla 3, y empleando como plataforma de análisis, los criterios de Seed et al. 1962 en: Djedid y Bekkouche 2000; Holtz et al. 1973 en: Djedid y Bekkouche 2001; Snethen 1980 en: Djedid

\begin{tabular}{|c|c|}
\hline Parámetro & Referencia \\
\hline Contenido de arcilla & Building Research Establishment 1980 en: Djedid y Bekkouche 2001 \\
\hline Índice de plasticidad & Building Research Establishment 1980 en: Djedid y Bekkouche 2001 \\
\hline Límite líquido & González de Vallejo et al. 2002 \\
\hline
\end{tabular}

Tabla 1. Criterios de diferentes autores utilizados en la clasificación de los mapas temáticos.

Table 1. Classification criteria of different authors used in the classification of thematic maps. 
Chinthaka-Ganepola, G.A., et al. Evaluación de la expansividad de los suelos en el... Boletín Geológico y Minero, 127 (4): $807-821$

\begin{tabular}{|c|c|c|c|}
\hline Contenido de arcilla & Índice de plasticidad & Límite líquido & Potencial de expansión final \\
\hline Bajo & Bajo a medio & Bajo a medio & Bajo a medio \\
\hline Bajo & Bajo & Bajo & Bajo \\
\hline Bajo & Bajo a medio & Bajo & Bajo a medio \\
\hline Bajo a medio & Bajo a medio & Bajo a medio & Bajo \\
\hline Bajo & Bajo & Bajo a medio & Bajo a medio \\
\hline Bajo a medio & Medio a alto & Bajo a medio & Bajo a medio \\
\hline Bajo a medio & Bajo a medio & Bajo & Medio a alto \\
\hline Bajo a medio & Medio a alto & Medio a alto & Bajo \\
\hline Bajo a medio & Bajo & Bajo & \\
\hline
\end{tabular}

Tabla 2. Matriz de las combinaciones para los diferentes niveles de potencial a la expansión.

Table 2. Combination matrix of different levels of potential expansiveness.

y Bekkouche 2001 y Building Research Establishment 1980 en: Djedid y Bekkouche 2001, se puede concluir, como primera aproximación al potencial de expansión de los suelos de reparto Hermanos Cruz, que estos presentan un potencial medio. Evidentemente, esto no significa que puntualmente, existan sitios dentro del área objeto de estudio, con potencial de expansión alto o bajo.

Como aplicación del segundo método (juego de curvas, ábacos), se plotearon los valores de índice de plasticidad y límite líquido en la carta de plasticidad de Casagrande (Figura 3 ). En este caso los valores se encuentran por encima de la línea $A$ en forma de banda, con la mayoría de los valores concentrados en la zona de las arcillas de baja plasticidad (CL), lo que sugiere según la literatura (González de Vallejo et al. 2002; Sowers y Sowers 1987) un potencial de expansión medio.

Posteriormente se utilizó el gráfico propuesto por Seed et al. (1960) en: Lucian (2006) para la evaluación del grado de expansión (Figura 4). En este juego de curvas, la mayoría de los puntos se ubican en la zona de potencial a la expansión medio. La actividad de los suelos comprende los valores entre 0.5 al 1.0, indicando la presencia de los suelos activos.

Como se muestra en la Tabla 3 y en las Figuras 3 y 4 , en la evaluación primaria del potencial de expansión de los suelos en el reparto Hermanos Cruz

\begin{tabular}{|l|c|c|c|c|}
\hline & $\begin{array}{c}\text { Límite líquido (LL) } \\
\%\end{array}$ & $\begin{array}{c}\text { Índice de plasticidad (IP) } \\
\%\end{array}$ & $\begin{array}{c}\text { Límite de retracción (LC) } \\
\%\end{array}$ & $\begin{array}{c}\text { Contenido de arcilla } \\
\%\end{array}$ \\
\hline No. de valores & 942 & 942 & 127 & 942 \\
\hline Mínimo & 14 & 1 & -0.1 & 0 \\
\hline 0,25 Percentil & 30 & 14 & 1.07 & 21 \\
\hline Mediana & 36 & 18 & 2 & 38 \\
\hline 0,75 Percentil & 44 & 25.25 & 12 & 84 \\
\hline Máximo & 88 & 62 & 20 & 29.737 \\
\hline Media & 37.793 & 20.585 & 1.07 & 28 \\
\hline Moda & 36 & 18 & 0.449 & 0.345 \\
\hline Asimetría & 1.008 & 0.959 & -1.298 & 0.183 \\
\hline Curtosis & 1.002 & 0.902 & 0.924 & 0.476 \\
\hline $\begin{array}{l}\text { Coeficiente de } \\
\text { variación }\end{array}$ & 0.308 & 0.445 & & \\
\hline
\end{tabular}

Tabla 3. Análisis estadístico de las propiedades índice.

Table 3. Statistical analysis of the index properties. 


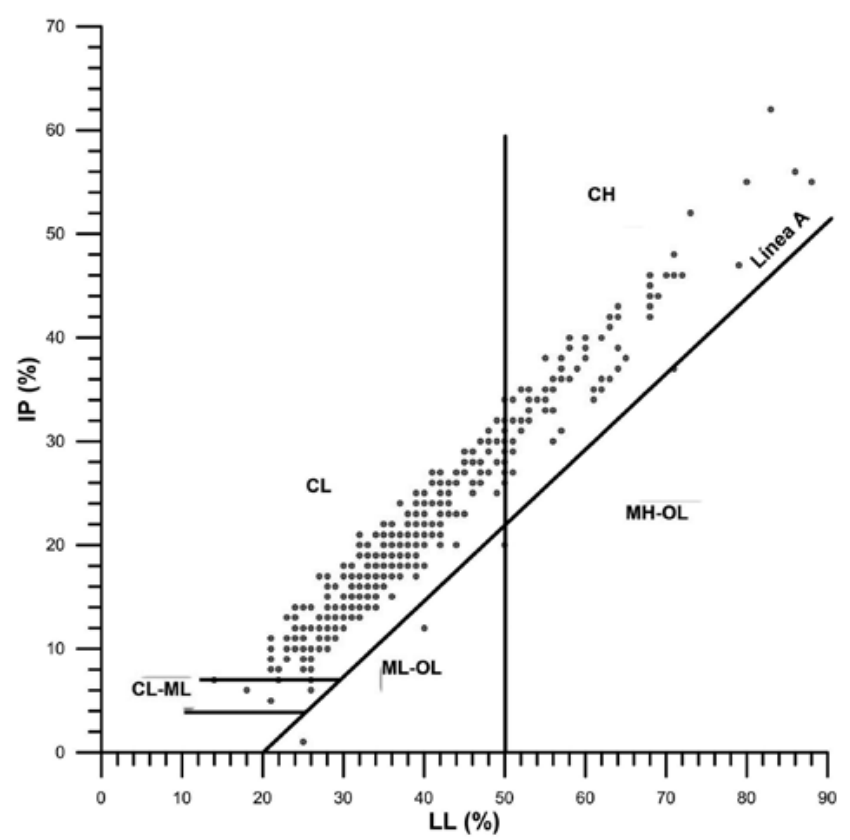

Figura 3. Representación de los valores de límite líquido e índice de plasticidad en la carta de plasticidad de Casagrande.

Figure 3. Plot of values of liquid limit and plasticity index in the Casagrande plasticity chart.

se incluyó el total de muestras de suelo (942), independientemente de su contenido de arcilla. Con este resultado se obtendría una visión general de la posible susceptibilidad de los suelos en el área a la expansividad.

Al mostrar estos suelos un potencial de expansión medio según los métodos aplicados anteriormente, se puede inferir la presencia de minerales arcillosos, como pueden ser la caolinita o la illita con una expansividad moderada. Algunos trabajos previos ya han reportado la presencia de caolinita (Girbert 2015).

Culminando la evaluación primaria del potencial de expansión de los suelos en el reparto Hermanos Cruz, se emplearon ecuaciones empíricas para pronosticar el hinchamiento libre (hl) (Ecuación I) e hinchamiento controlado (hc) (Ecuación II) (Delgado 2003). En ambas ecuaciones se empleó el valor promedio del índice de consistencia:

$$
\left[\mathrm{I}_{\mathrm{c}}=\frac{\text { Límite líquido }- \text { Humedad natural }}{\text { Índice de plasticidad }}\right]
$$

y de la actividad:

$\left[\mathrm{A}=\frac{\text { Índice de plasticidad }}{\% \text { en peso de material de tamaño de partículas menores de } 0.002 \mathrm{~mm}}\right]$

$$
\text { (Ic }=1.089 \% \text { y } A=0.754) \text {. }
$$

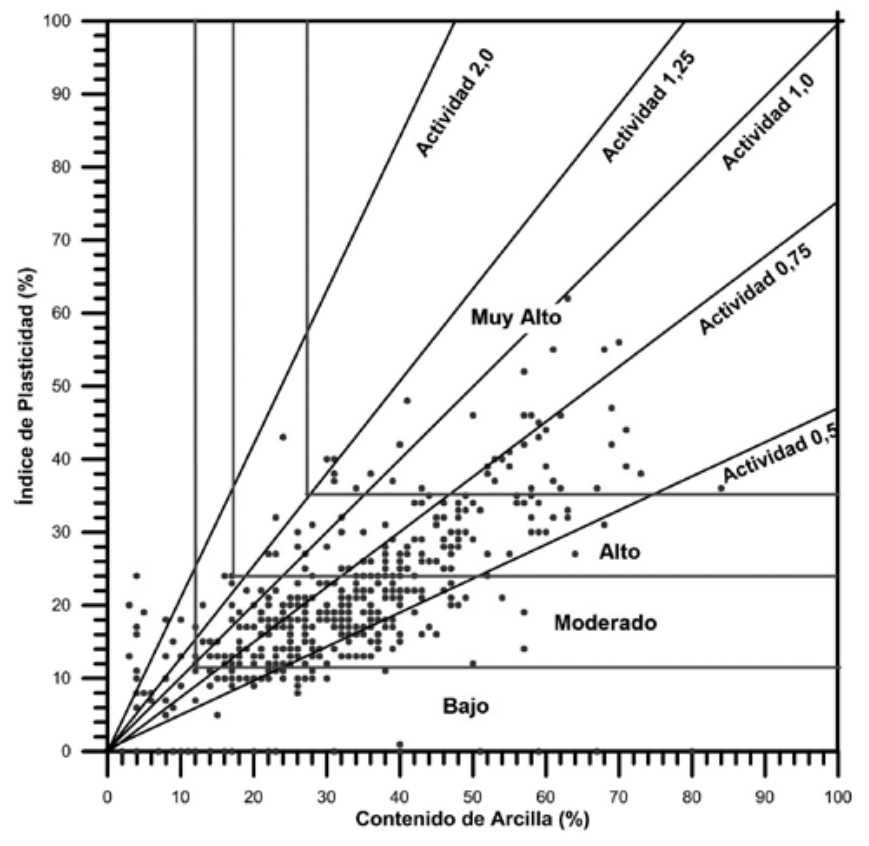

Figura 4. Representación de los valores en el gráfico propuesto por Seed et al. (1960) en: Lucian (2006).

Figure 4. Plot of values on the chart proposed by Seed et al. (1960) in: Lucian (2006).

$$
\begin{aligned}
& \log h \mathrm{~h}=0.54 \log \mathrm{A}+0.67 \mathrm{lc}-0.39 \\
& \mathrm{hl}=17.5 \% \\
& \log \mathrm{hc}=0.66+1.22 \log \mathrm{hl} \\
& \mathrm{hc}=150.5 \mathrm{KPa}
\end{aligned}
$$

Según la clasificación del grado de expansión propuesto por Delgado (2003) (Tabla 4), los suelos posen un potencial de expansión medio.

En este sentido, también se utilizó la ecuación empírica desarrollada por Martínez (2012) (Ecuación III) para determinar la actividad $A$, ecuación válida solo para valores de cohesión $\mathrm{C}$ menores que $70 \mathrm{KPa}$ :

\begin{tabular}{|c|c|c|}
\hline $\begin{array}{c}\text { Hinchamiento } \\
\text { libre (hl) } \\
\%\end{array}$ & $\begin{array}{c}\text { Hinchamiento } \\
\text { controlado (hc) } \\
\text { KPa }\end{array}$ & $\begin{array}{c}\text { Potencial de } \\
\text { expansión }\end{array}$ \\
\hline$<4$ & $<25$ & nulo \\
\hline $4-10$ & $25-75$ & bajo \\
\hline $10-22$ & $75-200$ & medio \\
\hline$>22$ & $>200$ & alto \\
\hline
\end{tabular}

Tabla 4. Clasificación del potencial de expansión según hinchamiento libre (hl) e hinchamiento controlado (hc) (Delgado 2003). Table 4.Classification of the potential expansiveness according to swelling potential (hl) and swelling pressure (hc) (Delgado 2003). 
$\mathrm{A}=0.70-0.0047$ (Arcillas, \%)+0.327 (Relación de vacíos) -0.0001 (C,KPa)

obteniéndose un valor promedio de actividad de 1.16 , indicando la presencia de los suelos activos.

Los resultados obtenidos a partir de los métodos aplicados anteriormente son muy similares y se puede concluir como primera aproximación, que los suelos del reparto Hermanos Cruz poseen un potencial de expansión "moderado". Los métodos aplicados hasta aquí no revelan el comportamiento espacial de los cambios volumétricos. Para ello es de gran importancia aplicar las técnicas de modelación de datos geotécnicos, principalmente en tercera dimensión. A continuación se presenta los resultados obtenidos a partir del análisis espacial de las propiedades de los suelos, así como los mapas de potencial de expansión a diferentes intervalos de profundidad de interés constructivo.

\section{Modelación de propiedades de los suelos}

El éxito en la estimación espacial del valor de una propiedad geotécnica, depende en gran medida de la correcta aplicación de los criterios geológicos. Para lograr una aproximación lo más realista posible, en este caso de estudio se agruparon los suelos en "tipos ingeniero-geológicos" los cuales deben representar unidades geológicas que posean comportamiento geotécnico similar. La estimación de cada propiedad del suelo estará limitada geométricamente en el espacio por estas unidades.

Los cinco tipos ingeniero-geológicos establecidos, en orden estratigráfico (desde la superficie hacia profundidad) según el análisis estadístico realizado con las bases de datos son:

- Capa vegetal/Relleno: Son los depósitos más superficiales que se encuentra en el área de estudio. Se tomaron en cuenta solo cuando su espesor supera los $0.75 \mathrm{~m}$.

- Aluvial marino grueso: Son suelos constituidos por arenas, arenas arcillosas y gravas arenosas que clasifican según el Sistema Unificado de Suelos (SUCS) como arenas arcillosas (SC) y gravas arcillosas (GC). Coincide con la fase arenosa de la Formación Guane.

- Aluvial marino fino: Suelos constituidos principalmente por alto contenido de finos según el SUCS clasifican como arcillas de alta plasticidad $(\mathrm{CH})$ y arcillas de baja plasticidad (CL). Coincide con la fase arcillosa de la Formación Guane.

- Eluvial: Son suelos o rocas blandas que pertenecen a la parte superior de la Formación Paso Real.
Está compuesta por arcillas carbonatadas con fragmentos de calizas. Fundamentalmente se clasifican como suelos areno arcillosos (SC) y arcillas de baja plasticidad (CL).

- Roca: Es la secuencia que conforma la parte basal del área de estudio. Está compuesta por alternancias de calizas y margas.

Utilizando este orden estratigráfico y la información en las bases de datos provenientes de 655 perforaciones, se generó el modelo geológico en 3-D del área de estudio (Figura 5). El modelo geológico brinda una aproximación de la distribución espacial de los tipos ingeniero geológicos. Los tipos ingeniero geológicos aluvial marino grueso y aluvial marino fino, correspondientes a la Formación Guane, son los que afloran con mayor extensión y al mismo tiempo los más estudiados geotécnicamente en el reparto Hermanos Cruz. En ellos generalmente abundan suelos arcillosos de baja y alta plasticidad y arenas arcillosas (CL, $\mathrm{CH}$ y $\mathrm{SC})$, con predominio de la fracción fina.

Posteriormente se elabora el modelo geomecánico (estimación de cada propiedad geotécnica dentro de la unidad geológica correspondiente).

Para ello, primero se hizo el análisis exploratorio de datos. Los histogramas confeccionados para las propiedades índices analizadas mostraron una distribución simétrica de los datos (Figura 6), lo que permitió aplicar los métodos de la geoestadística lineal (kriging ordinario) para la estimación espacial de cada variable. En la Figura 7 se muestran los variogramas multidireccionales que se utilizaron en la modelación de datos.

Del estudio de los variogramas se obtuvieron los modelos matemáticos que caracterizan la variabilidad espacial de las propiedades: contenido de arciIla, índice de plasticidad y límite líquido; los cuales intervienen directamente en la evaluación del potencial de expansión (Tabla 5). La estimación se limitó a los primeros $10 \mathrm{~m}$ del corte (los más superficiales), donde se concentra la mayor cantidad de muestras.

Como generalidad los semivariogramas direccionales no presentaron grandes diferencias entre sí, igual comportamiento presentó el semivariograma multidireccional, este análisis sugiere la no presencia (ausencia) de anisotropía en el plano X-Y.

Para finalizar la etapa de modelación de propiedades del suelo, se elaboran los modelos 3D del contenido de arcilla, el índice de plasticidad y el límite líquido (Figuras 8,9 y 10). Estos tres indicadores conforman la información primaria para los análisis integrados de datos, con el próposito de realizar análisis espaciales del potencial de expansión de los suelos 


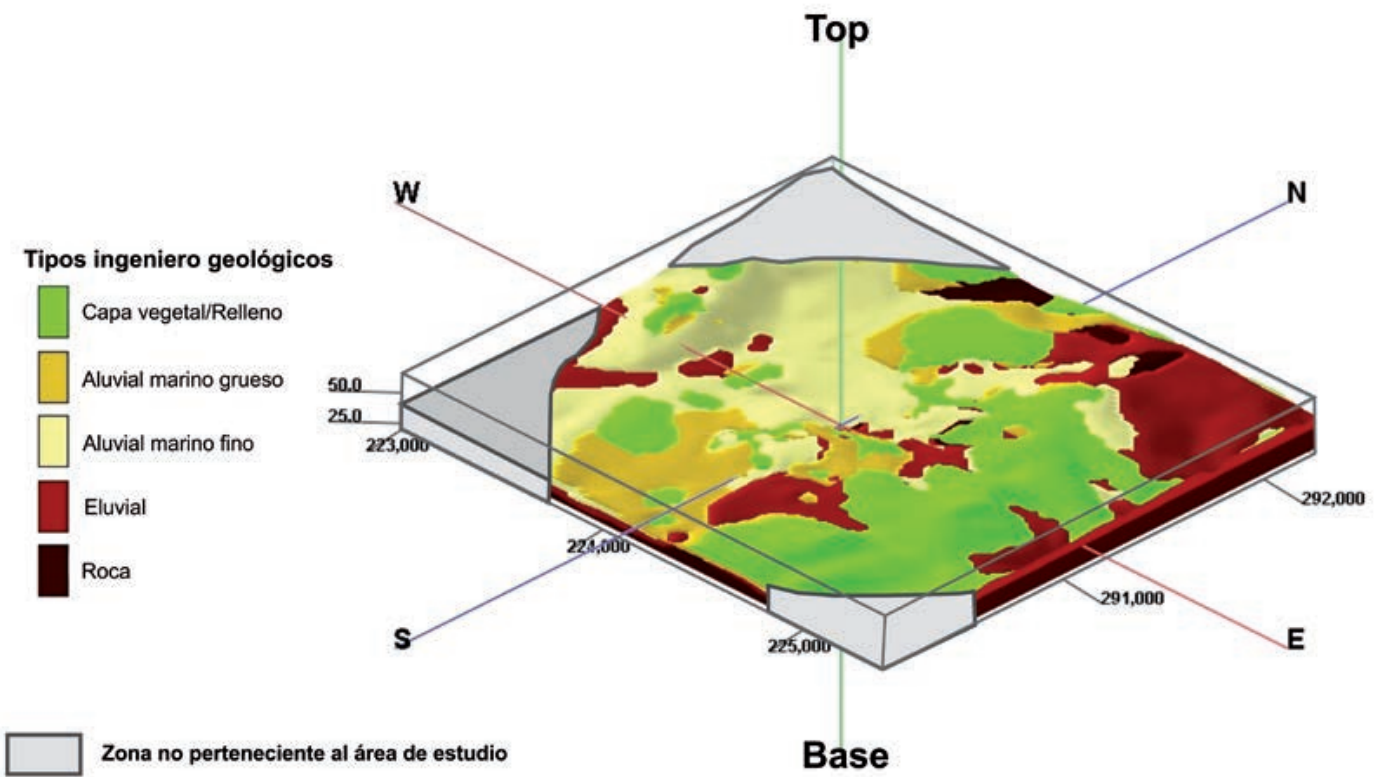

Figura 5. Modelo geológico mostrando la distribución espacial de los tipos ingeniero-geológicos. Resolución 25x25×1.

Figure 5. Geological model showing the spatial distribution of the engineering geological units. Resolution $25 \times 25 \times 1$.
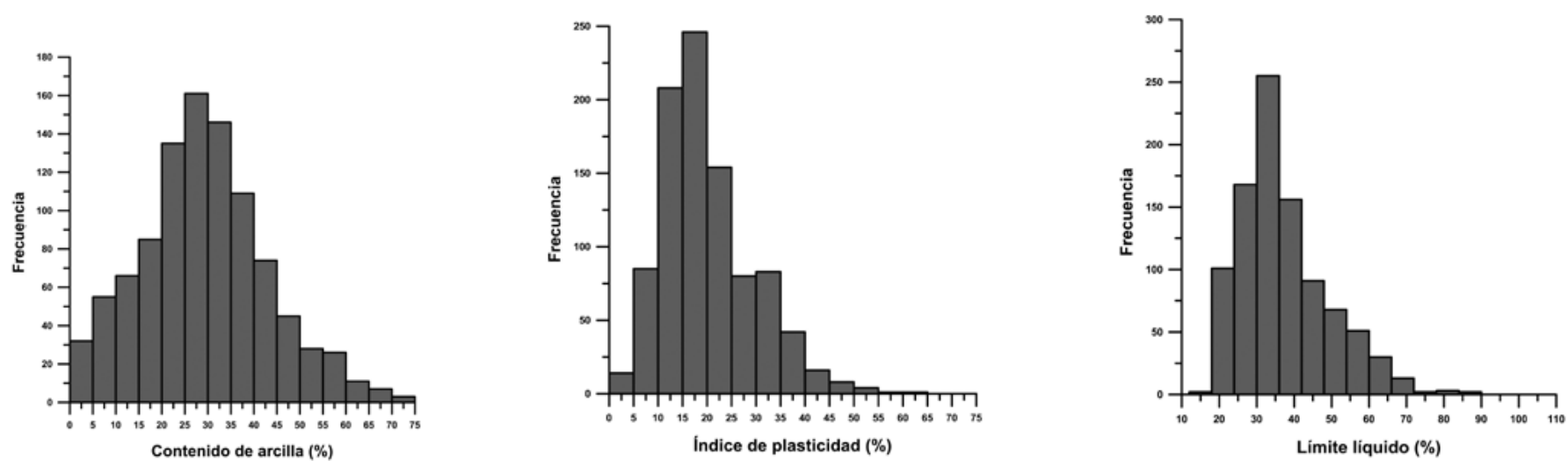

Figura 6. Histogramas obtenidos para las propiedades índice analizadas en el reparto Hermanos Cruz: contenido de arcilla, índice de plasticidad y límite líquido.

Figure 6. Histograms of the analyzed index properties of the Hermanos Cruz neighbourhood: clay content, plasticity index and liquid limit.
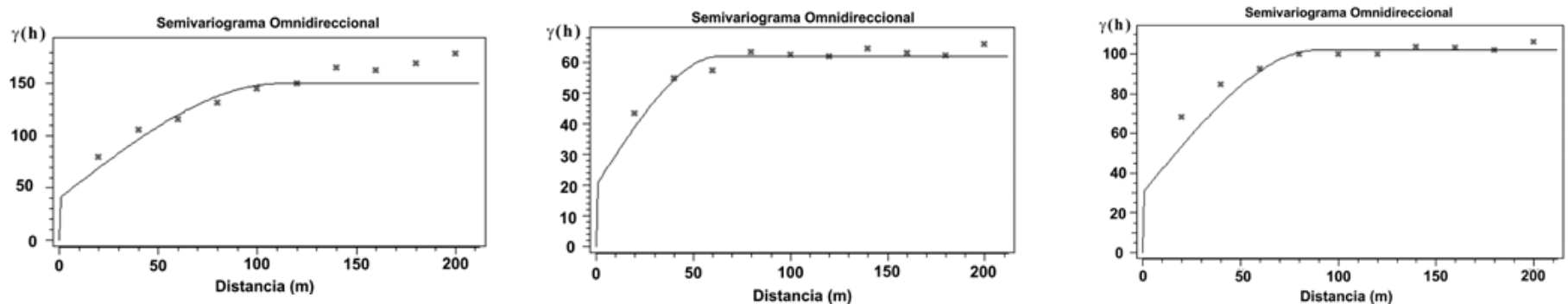

Figura 7. Variogramas multidireccionales obtenidos para las propiedades índice analizadas en el reparto Hermanos Cruz: contenido de arcilla, índice de plasticidad y límite líquido.

Figure 7. Omnidirectional variograms of the analyzed index properties of the Hermanos Cruz neighbourhood: clay content, plasticity index and liquid limit. 


\begin{tabular}{|c|c|}
\hline Propiedad geotécnica & Modelo matemático \\
\hline Contenido de arcilla & $\gamma(\mathrm{h})=40+110 \mathrm{sph}(112,110,2.5)$ \\
\hline Índice de plasticidad & $\gamma(\mathrm{h})=20+50 \mathrm{sph}(122,114,2.5)$ \\
\hline Límite líquido & $\gamma(\mathrm{h})=30+80 \mathrm{sph}(102,102,2.5)$ \\
\hline
\end{tabular}

Tabla 5. Modelos de la variabilidad espacial utilizados en la estimación de las propiedades de los suelos.

Table 5. Spatial variability models employed in the estimation of soil properties.

sobre plataforma SIG. La estimación obtenida permite la puesta en práctica de diferentes criterios de evaluación y analisis como se constatará en el proximo acápite.

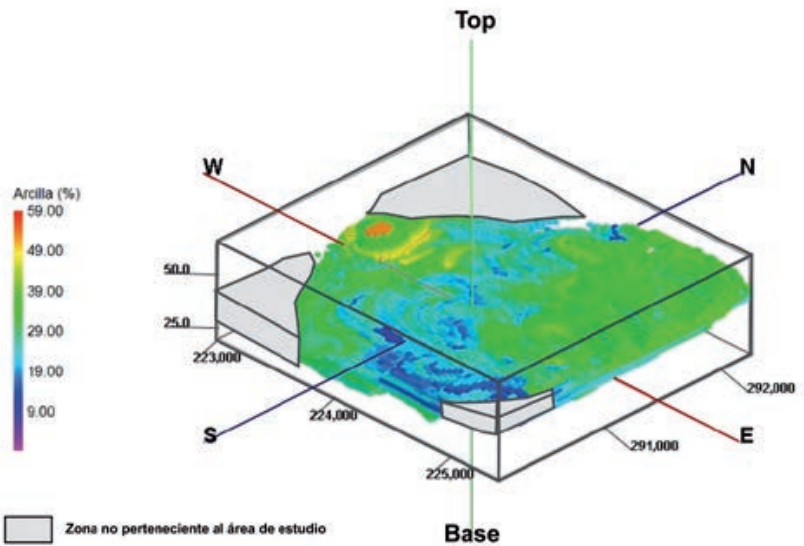

Figura 8. Modelo tridimensional del contenido de arcilla en los suelos del reparto Hermanos Cruz. Resolución 25x25x1.

Figure 8. Three dimensional model of the clay content. Hermanos Cruz neighbourhood. Resolution $25 \times 25 \times 1$.

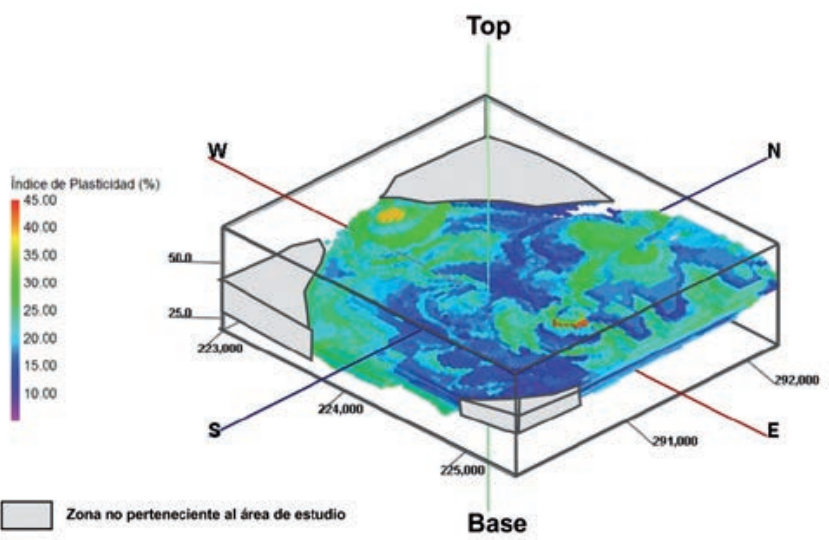

Figura 9. Modelo tridimensional del índice de plasticidad en los suelos del reparto Hermanos Cruz. Resolución 25x25×1.

Figure 9. Three dimensional model of the plasticity index. Hermanos Cruz neighbourhood. Resolution 25x25x1.

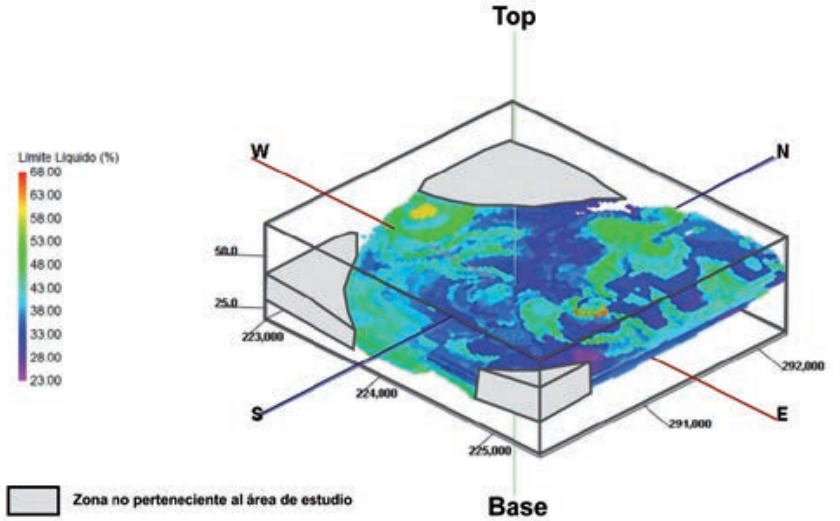

Figura 10. Modelo tridimensional del límite líquido en los suelos del reparto Hermanos Cruz. Resolución 25x25x1.

Figure 10. Three dimensional model of the plasticity index. Hermanos Cruz neighbourhood. Resolution 25x25x1.

\section{Representación cartográfica del potencial de expan- sión de los suelos sobre plataforma SIG}

Se presentan los mapas de potencial a la expansión de los suelos para tres niveles de profundidad. Los intervalos de profundidad analizados son de 0 a 1.0 $\mathrm{m}, 1.0$ a $2.0 \mathrm{~m}$ y de 2.0 a $3.0 \mathrm{~m}$, estas profundidades son las de mayor importancia para las cimentaciones superficiales.

De acuerdo a los criterios: Building Research Establisment (1980) en: Djedid y Bekkouche (2001) y González de Vallejo et al. (2002) sobre el potencial de expansión, se identificaron en el reparto Hermanos Cruz, tres niveles de potencial a la expansión de los suelos: bajo, bajo a medio y medio a alto. En la Tabla 6 se muestra los rangos que debe presentar cada una de las variables analizadas (contenido de arcilla, índice de plasticidad y límite líquido) para clasificar en cada nivel de expansión.

Los mapas temáticos indicativos de cada propiedad del suelo se reclasificaron de acuerdo a los rangos sugeridos en la Tabla 6 y se combinaron sobre plataforma de sistema de información geográfica 


\begin{tabular}{|l|c|c|c|}
\hline \multicolumn{1}{|c|}{ Potencial de expansión } & Contenido de arcilla & Índice de plasticidad & Límite líquido \\
\hline Bajo & $<30 \%$ & $<15 \%$ & $<30 \%$ \\
\hline Bajo a medio & $30 \%-60 \%$ & $15 \%-30 \%$ & $30 \%-50 \%$ \\
\hline Medio a alto & $60 \%-70 \%$ & $30 \%-45 \%$ & $50 \%-70 \%$ \\
\hline
\end{tabular}

Tabla 6. Clasificación del potencial de expansión de los suelos.

Table 6. Classification of the potential expansiveness of soils.

para obtener los mapas de potencial de expansión de los suelos para los tres niveles de profundidad anteriormente expuestos.

La cartografía obtenida muestra la existencia de sectores aislados, donde sus suelos clasificarían con un potencial de expansión de medio a alto. En estos sectores la columna de suelo está conformada predominantemente por suelos arcillosos que clasifican en el Sistema Unificado de Clasificación de Suelos (SUCS) como arcillas de alta y baja plasticidad $(\mathrm{CH}$ y CL). Uno de estos sectores (el de mayores dimensiones) se encuentra situado en la zona oeste del reparto Hermanos Cruz y es señalada con la letra " $\mathrm{H}$ " (coincide con el emplazamiento de un hospital) (Figuras 11, 12 y 13).

La mayor parte del área de estudio se caracteriza por suelos con un potencial de expansión de bajo a medio. Se puede apreciar que la dimensión de los sectores identificados con un potencial de medio a alto, disminuye con la profundidad. La mayor parte de estos suelos (con potencial de medio a alto), pertenecen al tipo ingeniero geológico "Aluvial marino

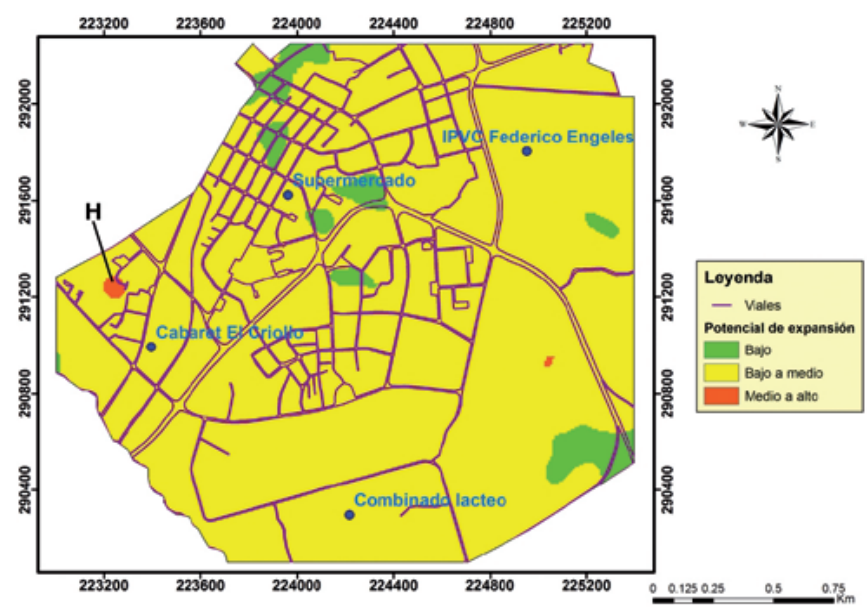

Figura 12. Mapa de potencial de expansión de los suelos en la zona de profundidad 1-2 m para el reparto Hermanos Cruz. Escala original 1:25000.

Figure 12. Map of the potential expansiveness of soils in the depth zone 1-2 m, Hermanos Cruz neighbourhood. Scale 1:25000.

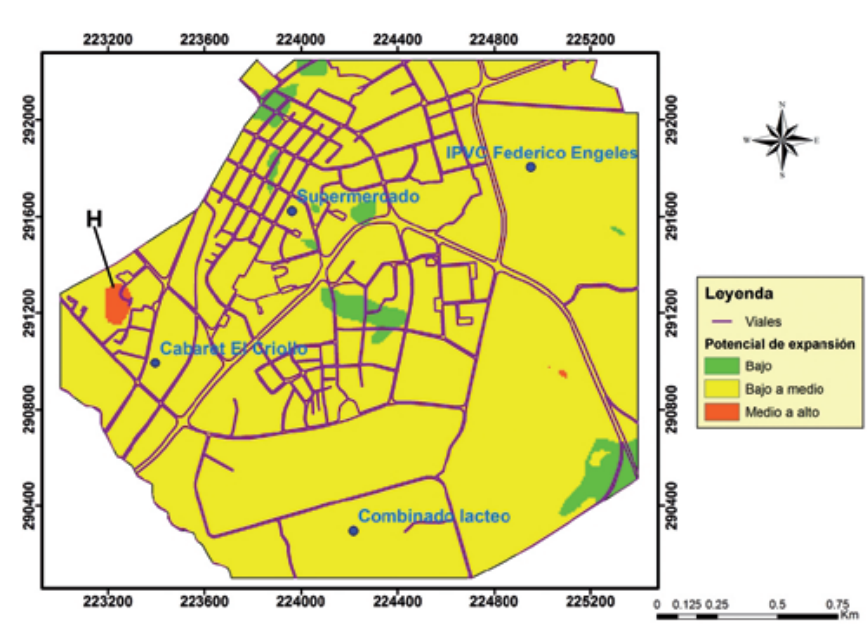

Figura 11. Mapa de potencial de expansión de los suelos en la zona de profundidad 0-1 m para el reparto Hermanos Cruz. Escala original 1: 25000.

Figure 11. Map of the potential expansiveness of soils in the depth zone 0-1 m, Hermanos Cruz neighbourhood. Scale 1:25000.

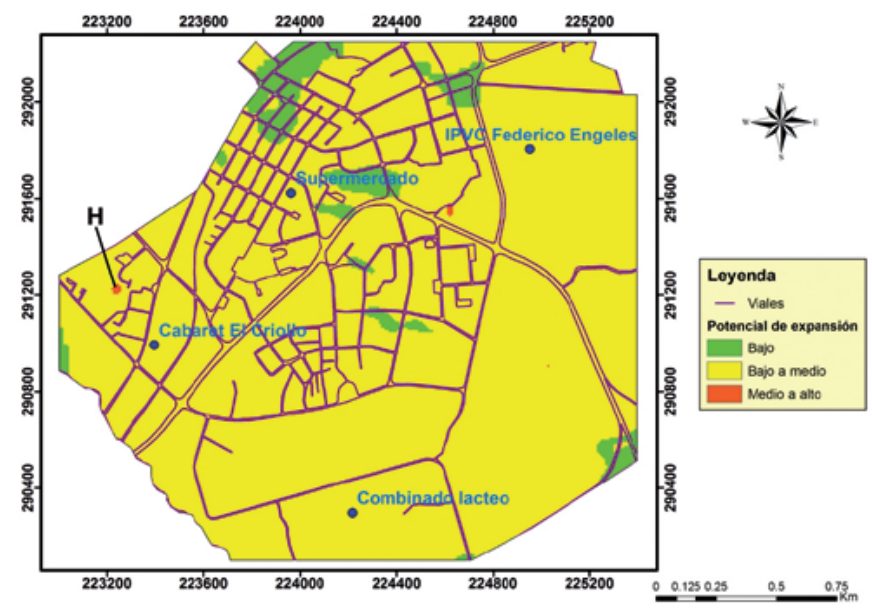

Figura 13. Mapa de potencial de expansión de los suelos en la zona de profundidad 2-3 m para el reparto Hermanos Cruz. Escala original 1:25000.

Figure 13. Map of the potential expansiveness of soils in the depth zone 2-3 m, Hermanos Cruz neighbourhood. Scale 1:25000. 
fino" asociado genéticamente a la Formación Guane y con menos frecuencia pueden pertenecer a los tipos ingeniero geológicos "Aluvial marino grueso" también de la Formación Guane y "Eluvial" de la Formación Paso Real.

Finalmente los mapas de potencial de expansión fueron sometidos a un proceso de validación, el análisis se realizó a partir de la superposición del mapa de potencial de expansión sobre el mapa de los daños por expansión de los suelos. De esta forma se pudo comprobar las coincidencias espaciales entre la ubicación de los daños con las zonas de pronósticos. En la Figura 14 se muestra, a modo de ejemplo, el mapa de potencial de expansión de los suelos en la zona de profundidad 0-1 m, sobre el cual se superponen las afectaciones a las construcciones más importantes identificadas en el área de estudio. Este resultado sugiere la toma de medidas ingenieriles correctivas en estos sitios, especialmente en los casos de construcciones ligeras emplazadas en suelos con pronóstico de potencial a la expansión de medio a alto.

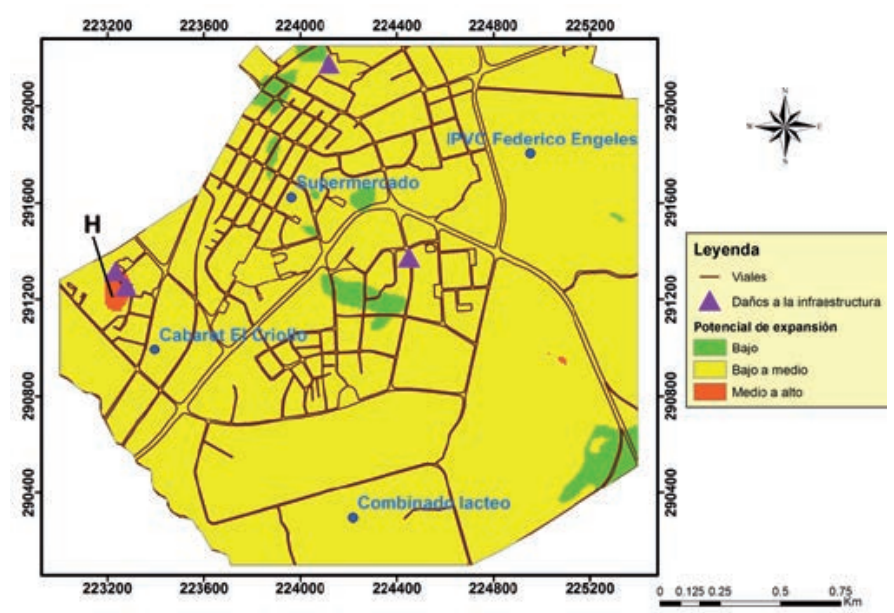

Figura 14. Distribución de afectaciones con diagnóstico de presencia de arcillas expansivas y su relación con el mapa de potencial de expansión de los suelos en la zona de profundidad 0-1 m.

Figure 14. Spatial distribution of the damage to infrastructure due to expansive soils and its relationship with the map of the potential expansiveness of soils in the depth zone 0-1 $\mathrm{m}$.

\section{Conclusiones}

1. El empleo de curvas y ecuaciones empíricas permiten una evaluación preliminar del potencial de expansión de los suelos en el reparto Hermanos Cruz, que mostraron un grado de expansión "moderado" de los suelos, pero no permitieron revelar el comportamiento espacial de los cambios volumétricos.
2. El modelo geológico del substrato sobre el que está edificado el reparto Hermanos Cruz, está definido por la presencia de cinco tipos ingeniero-geológicos: Capa vegetal-Relleno, Aluvial marino grueso, Aluvial marino fino, Eluvial y Rocoso. Estas unidades se identifican como zonas de comportamiento homogéneo en términos de geotecnia.

3. La integración de los resultados de la modelación de las propiedades geotécnicas junto con los métodos cuantitativos, permitieron generar mapas de potencial de expansión de los suelos en diferentes zonas de profundidad sobre plataforma SIG. Esta información es de vital importancia en las tareas de planificación urbana del reparto Hermanos Cruz, pues sirven de guía en la realización de los proyectos constructivos con mayor seguridad.

4. La aplicación de este procedimiento, reveló que los suelos del reparto Hermanos Cruz poseen un potencial de expansión bajo a medio y la existencia de sectores aislados con un potencial de expansión medio a alto.

\section{Referencias}

Bimbela, C.A. 2014. Solución ecológica y sustentable para la disminución de expansión en arcillas mediante perforaciones. Tesis de Maestría, Facultad de Ingeniería, Universidad Autónoma de Querétaro, México, 84 pp.

Bourgine, B., Dominique, S., Marache, A. and Thierry, P. 2009. Tools and methods for constructing 3D geological models in the urban environment: the case of Bordeaux. In: Culshaw, M. G., Reeves, H. J., Jefferson, I. \& Spink, T. W. (ed.), Engineering Geology for Tomorrow's Cities. Engineering Geology Special Publications, Geological Society, London.

Breysse, D., Piette, C., Marache, A. and Fabre, R. 2009. Data analysis and geotechnical properties modeling of urban soils. In: Culshaw, M. G., Reeves, H. J., Jefferson, I. \& Spink, T. W. (ed.), Engineering Geology for Tomorrow's Cities. Engineering Geology Special Publications, Geological Society, London.

Cabrera, J., Guardado, R., Peláez, R. y González, N. 2004. Regionalización hidrogeológica de la provincia de Pinar del Río en un SIG. Minería y Geología 20 (1-2): 24-31.

Choi, Y. and Park, H. 2009. Integrating GIS and 3D geostatitical methods for geotecnical characterization of soil properties. In: Culshaw, M. G., Reeves, H. J., Jefferson, I. \& Spink, T. W. (ed.), Engineering Geology for Tomorrow's Cities. Engineering Geology Special Publications, Geological Society, London.

Cuador, J. Q., Quintero, A., Estévez, E. y Ramírez, R. 1997. Elementos de Geoestadística. Informe inédito. Universidad de Pinar del Río, Pinar del Río, 40 págs.

Delgado, D. E. 2003. Estudio del comportamiento de los suelos cohesivos con problemas especiales de 
inestabilidad volumétrica y sus soluciones ingenieriles. Tesis doctoral. Departamento de Ingeniería Civil, Universidad de Central Marta Abreu de Las Villas, Santa Clara, Cuba 150 pp.

Djedid, A., Bekkouche, A. and Aissa, S.M. 2001. Identification and prediction of the swelling behavior of some soils from the Tlemcen region of Algeria. Bulletin des laboratoires des ponts et chausses, 233, 69-77.

Girbert, Y. 2014. Pronóstico sobre la expansividad de las arcillas en la ciudad de Pinar del Río. Tesis de Maestría, Departamento de Geología, Universidad de Pinar del Río, Cuba, 100 pp.

González de Vallejo, L.I., Ferrer, M., Ortuño, L. y Oteo, C. 2002. Ingeniería Geológica. Pearson Educación. Madrid, $712 \mathrm{pp}$.

Jones, L.D. and Terrington, R. 2011. Modelling volume change potential in the London clay. Quarterly Journal of Engineering Geology and Hydrogeology, 44, 109-122.

Lucian, C. 2006. Geotechnical aspects of buildings on expansive soils in Kibaha, Tanzania. Tesis doctoral. Departamento de Ingeniería Civil y Arquitectura, Royal Institute of Technology, Stockholm, Suecia, 150.

Martinez, R. 2012. Determinación de forma empírica de la carga contra hinchamiento. Informe inédito. Archivo técnico de la Unidad de Investigaciones para la Construcción de Pinar del Río, Empresa Nacional de Investigaciones Aplicadas, Pinar del Río, 30 págs.

Mendes, R. M. and Lorandi, R. 2009. Indicator kriging geostatistical methodology applied to geotechnics project planning. In: Culshaw, M. G., Reeves, H. J., Jefferson, I. \& Spink, T. W. (ed.), Engineering Geology for Tomorrow's Cities. Engineering Geology Special Publications, Geological Society, London.

Ordaz, A., Estévez, E., Hernández, J.R. y Chuy, T.J. 2014. Modelación de parámetros geotécnicos como contribución a la zonación sísmica local: ciudad de San Cristobal, Cuba. Minería y Geología. 30 (1), 1-16.

Ordaz, A. 2014. Evaluación de las condiciones ingeniero-geológicas para el pronóstico de la respuesta dinámica de los suelos. Caso de estudio: Ciudad de San Cristóbal, Cuba occidental. Tesis de Doctorado, Departamento de Geología, Universidad de Pinar del Río, Cuba, 171 pp.

Rivera, Z.C. 2013. Proceso metodológico para la modelación de los suelos. Caso de estudio: Ciudad de Santiago de Cuba. V Convención Cubana Ciencias de la Tierra, La Habana, 1-11.

Sowers, G.B. y Sowers, G.F. 1987. Introducción a la mecánica de suelos y cementaciones. Primera Parte. Editorial Revolucionaria, La Habana, 320 pp.

Recibido: mayo 2015

Revisado: julio 2015

Aceptado: agosto 2015

Publicado: diciembre 2016 
